\title{
Summary: Cognition in 2014
}

\author{
TERRENCE J. SEJNOWSKI ${ }^{1,2}$ \\ ${ }^{1}$ Howard Hughes Medical Institute, the Salk Institute for Biological Studies, La Jolla, California 92037 \\ ${ }^{2}$ Division of Biological Sciences, University of California at San Diego, La Jolla, California 92093 \\ Correspondence: terry@salk.edu
}

\begin{abstract}
The goal of the 79th Cold Spring Harbor Symposia on Quantitative Biology on Cognition held on May 28-June 2, 2014 was to survey recent advances in cognitive neuroscience and assess future prospects. The symposium succeeded beyond the dreams of the organizers and the participants were treated to an extraordinarily rich feast of 58 long talks, six short talks, and 137 posters. Equally important to the success of the symposium was the perfect setting for informal scientific exchange between 260 participants generously provided by the Cold Spring Harbor Laboratory. The sense that emerged from the symposium was that a threshold had been crossed and a new era in the study of cognition was underway. My attempt here will be to capture that sense of awakening, to trace the strands that gave rise to it, and to access its implications for future discoveries.
\end{abstract}

\section{WHAT HAS CHANGED?}

An earlier Cold Spring Harbor Symposia on The Brain in 1990 covered a much broader range of topics that included molecular mechanisms for signaling, neural development, and sensory and motor systems, as well as cognitive neuroscience. The sweep of the meeting reflected the emergence of neuroscience as a young but vibrant discipline and in particular the impact of molecular biology on many of the difficult problems that previously had resisted explanations. As Mike Stryker, who gave the Summary at that meeting put it, "Is Biology Ready to Take on the Brain?" The meeting revealed how productive the interactions were between neuroscience and the rest of biology.

The papers on cognitive neuroscience from the 1990 symposium reflected the progress that had been made using single-unit recording techniques, which revealed the properties of single neurons, particularly in the visual system and hippocampus. The focus on single neurons dominated thinking in that era and studies using electroencephalogram and event-related potential had much poorer resolution. Brain imaging techniques were in their infancy, with pioneering work on positron emission tomography beginning at the University of Washington (Petersen et al. 1988) and the invention of functional magnetic resonance imaging (fMRI) at Bell Labs (Ogawa et al. 1990). Anatomical techniques for staining neurons and tract tracing were classical, some going back to Golgi and Ramón y Cajal in the 19th century. The molecular revolution that had already swept through other areas of neuroscience, the cloning of ion channels and gene expression during development, had not yet reached systems neuroscience.

Francis Crick (1979) wrote a prescient essay that foretold what the impact of techniques from molecular genetics would have on systems neuroscience:
For example, a method that would make it possible to inject one neuron with a substance that would then clearly stain all the neurons connected to it, and not others, would be invaluable. So would a method by which all neurons of just one type could be inactivated, leaving the other more or less unaltered.

And, indeed, this has come to pass, as reflected in major advances that have been made in the last several years using viruses (Wall et al. 2010) and optogenetics (Zhang et al. 2006). Our ability to target specific types of neurons, to optically record, stimulate, and suppress their activity, has greatly accelerated research because of both the quantity of data we can collect and the new types of experiments that can be done by manipulating neural systems with exquisite selectivity.

Another major shift since 1990 is that more laboratories are using advanced data analysis techniques and computational models to guide their research. Only a few talks at the 1990 Brain Symposium used computational approaches but more than half of the talks at the $2014 \mathrm{Cog}$ nition Symposium incorporated them. Here again, Crick (1979) had anticipated how theory and experiment might be integrated:
Hence an important role for theory in neurobiology is not merely trying to create correct and detailed theories of neural processes (which may be an extremely difficult task) but pointing to which features it would be most useful to study and in particular to measure, to see what kind of theory is needed.

We now have theoretical frameworks for how populations of neurons encode and store information, which are being used to analyze recordings from large-scale populations of neurons.

But on one prediction Crick (1979) missed the mark:

\footnotetext{
It is no use asking for the impossible, such as, say, the exact wiring diagram for a cubic millimeter of brain tissue and the way all its neurons are firing.
} 
What Crick failed to predict was the rapid advances in techniques for working out the wiring diagrams of neural circuits. John White and Sydney Brenner had laboriously traced out and manually reconstructed the wiring diagram for Caenorhabditis elegans from electron microscope cross sections, a bit of 21 st century science in the 20th century. Large-scale production of serial electron micrographs and machine learning have now made it feasible to automate and scale-up the reconstruction of ever-larger neural circuits. Similarly, advances in recording techniques have made it possible to record simultaneously from hundreds of neurons.

\section{WHERE ARE WE TODAY?}

New techniques (Fig. 1) have made it possible to probe with much higher precision than was previously possible how neural circuits give rise to complex behaviors. It is not possible to summarize all of the results that were presented at the Symposium here, but instead I have selected a few representative examples that illustrate what has been accomplished, arranged according to the spatial scale of the measurements.

\section{Genetically Encoded Indicators}

An elegant example of how sensory information is transformed into actions is found in C. elegans, which has a relatively short chain of neurons between sensory receptors and motoneurons. Neurons in C. elegans are small and difficult to record from with microelectrodes because of body turgor. But now activity patterns can be followed with genetically encoded calcium-sensitive dyes. Cornelia Bargmann showed that sensory neurons reliably responded to chemical signals, but the next neuron down the chain displayed response variability that was highly correlated with reversal behavior. As she put it, "One of the most reliable aspects of behavior is its unreliability." We can now pinpoint where the variability is introduced. The ability to probe all the neurons in the circuit and to manipulate as well as observe them is providing a powerful way to divine the logic of neural circuits.

\section{Genetic Fingerprints}

The brain has more different cell types than any other organ by several orders of magnitude and we are far from having a complete catalog. This is changing rapidly as techniques from molecular genetics are being used to identify cell types by their pattern of transcription factors, which regulate gene expression. Thomas Jessell showed how this can be done to distinguish more than a dozen types of inhibitory interneurons in the spinal cord, using advanced statistical techniques for sorting through many different combinations of transcription factors. Other sources of cell identity may come from the pattern of DNA methylation, which also regulates gene expression. The DNA in neurons has a special pattern of methylation not found in other organs of adults (Lister et al. 2013).

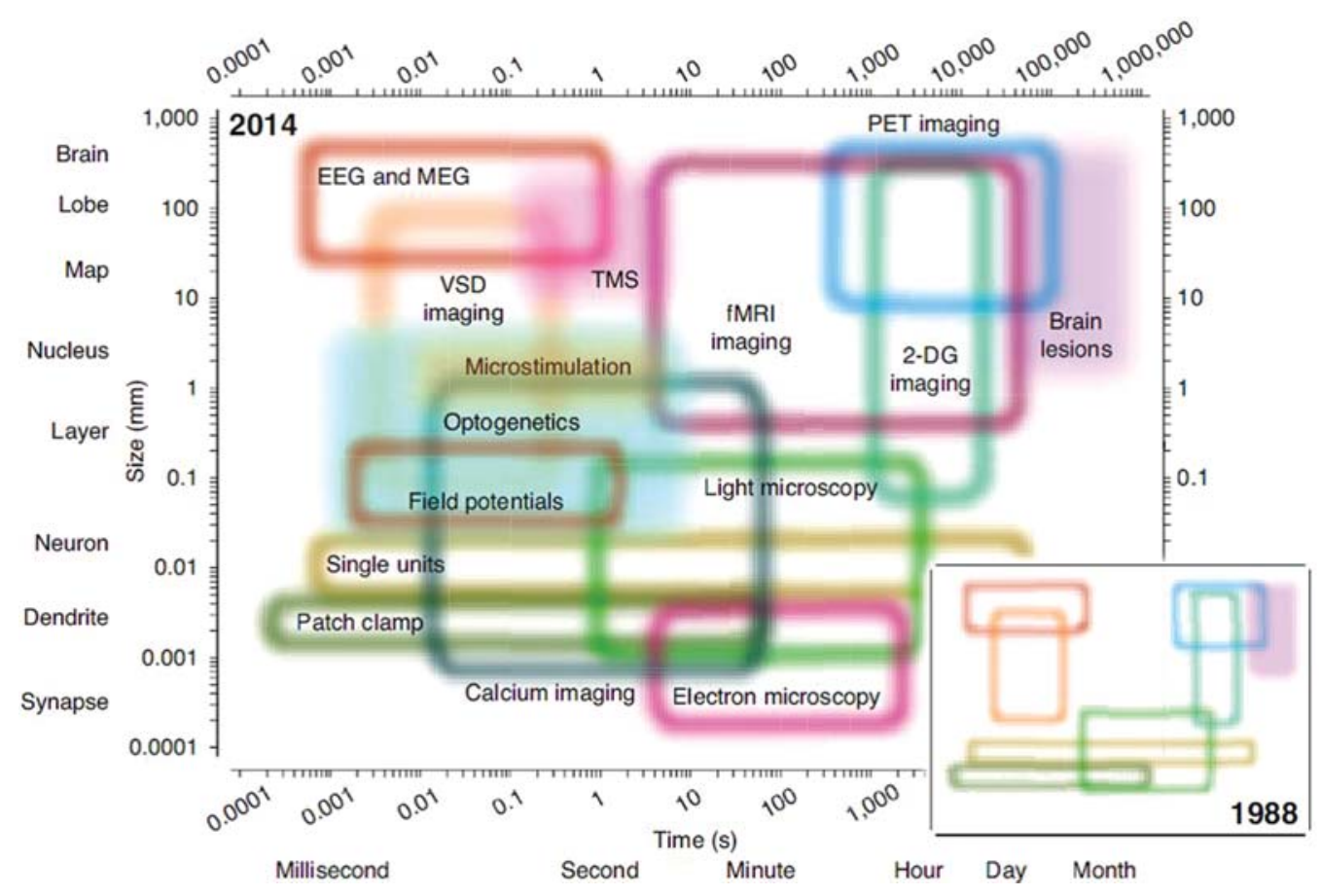

Figure 1. The space-time domain of methods in neuroscience in 2014, in contrast with a similar space-time map in 1988 (inset). Each colored region represents the useful domain of spatial and temporal resolution for one method available for the study of the brain. Open regions represent measurement techniques, filled regions, perturbation techniques. The gaping holes in the 1988 diagram were filled in with functional magnetic resonance imaging (fMRI), calcium imaging, optogenetics, and transcranial magnetic stimulation (TMS). (Reprinted, with permission, from Sejnowski et al. 2014.) 


\section{Neural Circuits}

Hassenstein and Reichardt (1956) proposed a simple neural circuit that could compute the direction of motion of a visual stimulus moving across a fly eye. Despite heroic efforts over the next 50 years progress was slow because of the complexity of the neuropil in the fly eye. In just the last few years, Alexander Borst, following in Reichardt's footsteps, used cell-specific optogenetic techniques and connectomic reconstruction to identify the components of the circuit. Nature has implemented a version of the Hassenstein and Reichardt model, separating the $\mathrm{ON}$ and OFF pathways, in essence devoting twice the machinery necessary to compute the direction of motion because of biophysical limitations. This shows how an apparently complex circuit may implement a relatively simple algorithm. It also illustrates how much progress can be made very quickly if the right tools are available to an experimenter to answer the question.

\section{Dynamic Stability}

Invertebrate neural systems such as the stomatogastric ganglion of the lobster were once considered "simpler systems," but it is now apparent that they have complex mechanisms that allow small numbers of neurons to generate a wide range of complex dynamical behaviors that are difficult to predict from the spatial distribution of dozens of types of nonlinear ion channels. Eve Marder asked how the observed robustness in behavior is possible given the heterogeneity of the underlying mechanisms. She showed that neuromodulators are a source of circuit stability. This important principle could also be demonstrated in a "simple neural model," which provides a framework for exploring why neuromodulation is so effective at stabilizing network properties.

\section{Large-Scale Recordings}

Much can be gained by recording from all the neurons in a circuit that controls a behavior. William Kristan recorded with voltage-sensitive dyes from many of the neurons in leech ganglia that are involved in the decision to swim or crawl. Some neurons are involved in both behaviors, with different patterns of activity. The switch between the two patterns occurs rapidly, in less than one swim cycle, which is a signature of what theorists have called "attractor" states that were analyzed in simple models of neural networks in the 1980s. Evidence for attractors has also been found in the hippocampus, where recurrent networks settle into states that represent different places in the environment (Colgin et al. 2010).

\section{Spike Timing Measurements}

We know a great deal about brain activity in response to sensory stimuli, but very little about internally generated brain activity that has neither a sensory nor a motor correlate. The discovery of replay in the hippocampus has given us a rare glimpse into what the brain does offline, when it is no longer in a closed loop with the sensory environment. Loren Frank reported that previously experienced patterns of activity in the hippocampus of awake rats are replayed and that disruption of the replay interferes with learning and memory-guided decision-making. Using large-scale recordings of hippocampal neurons, Györgi Buzsáki showed that these internally generated self-perpetuating sequences are supported by oscillations and precise spike timing. These discoveries open up a new window on internal brain states.

\section{Activity Tagging}

Psychologists have long postulated that human memory was encoded in the brain as an "engram" (Bruce 2001) and although many laboratories focusing on single neurons were able to make progress studying the mechanisms for synaptic plasticity, the engram remained an elusive concept; that is, until molecular tools became available to tag the neurons in an engram. Susumu Tonegawa told us about an experiment in which he was able to tag neurons involved in fear conditioning with channelrhodopsin-2, which allowed him to reinstate the engram by shining blue light on the tagged neurons (Redondo et al. 2014). Many other classical concepts based primarily on behavioral experiments can now be traced to specific neural circuits and brain systems.

\section{Language Development}

Language is a quintessential human behavior that develops rapidly during the first 2 years of life. Brain imaging has made it possible to follow the maturation of the cortical circuits responsible for language. Patricia Kuhl showed that structural MRI features can predict a baby's future language performance. Magnetoencephalography revealed that when infants listen to speech, brain activation occurs not only in auditory areas but also in brain regions involved in speech motor planning. This is evidence for a close collaboration between the sensory and motor systems in generating language, something that had been long suspected.

\section{Virtual Reality}

Computer games have become more popular than television in some age groups and it is of some concern to know how this affects the brain, especially of developing children. Daphne Bavelier reported that some games enhance sensory, attentional, and cognitive skills. These generalize beyond the specific skills learned in the games. Adam Gazzaley has taken this even further by showing how personalized close-loop virtual reality games can have therapeutic value and sustainable cognitive enhancement. This opens up a new window into human learning that could have major implications for education and rehabilitation.

\section{Thought}

One topic in cognition was notable by it absence at the symposium. At the 1990 Brain Symposium Francis Crick and Christof Koch presented "Some Reflections on Visual 
Awareness." No one did more to draw our attention to the problem of consciousness in the 20th century than Crick (Sejnowski 2015). The topic of consciousness never came up in the formal talks at the 2014 Cognition Symposium. Zachary Mainen, however, addressed free will in his talk, which concluded that brain recordings that are correlated with decisions may reflect evidence accumulation rather than the decision itself. As techniques get better for testing causality by manipulating patterns of activity in neural populations it may become possible for us to probe the mechanisms underlying thoughts and actions.

\section{WHERE WILL WE BE IN 2025?}

On 3 April 2013, President Obama announced the BRAIN Initiative, whose goal is to develop innovative neurotechnologies. The working group that advised the Director of the National Institutes of Health (NIH) for the BRAIN (Brain Research through Advancing Innovative Neurotechnologies) Initiative prepared a detailed plan and goals. The final report, which was accepted by the NIH on June 5, 2015 (BRAIN 2025: http://www.brain initiative.nih.gov/2025/index.htm), a few days after the symposium, identified seven major goals.

1. Discovering diversity: Identify and provide experimental access to the different brain cell types to determine their roles in health and disease.

2. Maps at multiple scales: Generate circuit diagrams that vary in resolution from synapses to the whole brain.

3. The brain in action: Produce a dynamic picture of the functioning brain by developing and applying improved methods for large-scale monitoring of neural activity.

4. Demonstrating causality: Link brain activity to behavior with precise interventional tools that change neural circuit dynamics.

5. Identifying fundamental principles: Produce conceptual foundations for understanding the biological basis of mental processes through development of new theoretical and data analysis tools.

6. Advancing human neuroscience: Develop innovative technologies to understand the human brain and treat its disorders; create and support integrated human brain research networks.

7. From BRAIN Initiative to the brain: Integrate new technological and conceptual approaches produced in Goals (1)-(6) to discover how dynamic patterns of neural activity are transformed into cognition, emotion, perception, and action in health and disease.

The NIH BRAIN Initiative and complementary programs at the National Science Foundation (NSF) and the Defense Advanced Research Projects Agency (DARPA) are under way and are accelerating the pace of innovation in cognitive neuroscience. The overall goal is to scale up current techniques so that by 2025 it will be possible to have connectomic wiring diagrams for all areas of the mouse brain, to be able to record simultaneously from a million neurons, and to develop sophisticated behavioral paradigms that engage multiple brain systems under more natural conditions. These advances will generate enormous data sets that will require new experimental designs, the development of new algorithms for analyzing the data, new ways to model the data, and new theories for interpreting the results (Sejnowski et al. 2014). Some of these data sets will be made available to the community, just as the availability of large genomic data sets, crystallographic data, and astronomical data from the Hubble Space Telescope have enhanced their usefulness.

All of these goals will depend on scaling up the resolution, speed, and comprehensiveness of behavioral, anatomical, physiological, and genetic techniques by factors of thousands. We are already on an exponential rise in the number of neurons that can be simultaneously recorded, with data sets that include calcium measurements from 1000 neurons in the hippocampus with 10msec resolution (Deisseroth and Schnitzer 2013) and 80,000 neurons in the zebrafish with 1 -sec resolution (Keller et al. 2015). Machine learning, which provides a set of powerful algorithms for analyzing data in highdimensional spaces, has made it possible to automatically track behavior with high spatial and temporal resolution (Anderson and Perona 2014). However, each algorithm makes statistical assumptions and care must be taken to confirm that they are satisfied by the brain data.

What cannot be predicted today is what impact the development of major new techniques could have on questions that we can ask about the brain. For example, at the symposium Tony Zador outlined a method for automating the collection of data from long-range tracts in the brain using short random sequences of RNA as barcodes, taking advantage of viruses to deliver the barcodes to neurons in one area and collect them in other brain areas. This would take advantage of advances in sequencing techniques to solve anatomical problems and could make it possible to rapidly, cheaply, and routinely obtain high-quality connectomic data. If this works it will revolutionize anatomical studies.

Another area where we can expect rapid progress to be made is in the study of human cognition. In many ways humans are the best model system to study humans. Imaging techniques allow us to observe human brain activity noninvasively and direct recording from the cortex and subcortical structures in patients with epilepsy are giving us new insights into language and memory consolidation. The current standards in human electrical recordings and deep brain stimulation is 30 years behind the state of the art in model systems such as monkeys and mice. The Food and Drug Administration, which approves drugs and devices, has a fast track for Investigational Device Exemptions (IDE), which should lead to a quantum leap in recording from and stimulation of human brains. DARPA has launched two major programs to develop better devices for closed loop recording and stimulation for treating neuropsychiatric disorders (SUBNET) and memory recovery (RAM).

Finally, animal models of human mental disorders and degenerative brain diseases have not translated into effec- 
tive drugs for treating humans. For example, drugs that have been developed at great expense for Alzheimer's disease based on mouse models have failed in clinical trials. There is thus a great need for transgenic nonhuman primate models of neurological diseases and neuropsychiatric disorders, which is already under way and should be widely available by 2025 (Belmonte et al. 2015). This could lead to a better understanding of these brain diseases and disorders at the circuit level and more precise ways to target the underlying dysfunctions.

\section{CONCLUSION}

A question came up in discussion with Bruce Stillman at the symposium picnic: "Is Biology Ready to Take on Cognition?" The conclusion from the 1990 Brain symposium was that biology was ready to take on the brain, and the question was whether biology was ready to take on the cognitive functions of the brain. What surprised me and others at the conference was the extent to which cognition has already become a part of biology and this will accelerate with the BRAIN Initiative. We can anticipate that within the near future tools and techniques will become available that will in time illuminate the biological mechanisms underlying language, thought, free will, and consciousness. A new neurotechnology industry will be needed to translate these advances into medical devices that can alleviate suffering from brain dysfunctions.

\section{REFERENCES}

Anderson DJ, Perona P. 2014. Toward a science of computational ethology. Neuron 84: 18-31.

Belmonte JC, Callaway EM, Churchland P, Caddick SJ, Feng G, Homanics GE, Lee KF, Leopold DA, Miller CT, Mitchell JF, et al. 2015. Brains, genes and primates. Neuron 86: 617-631.
Bruce D. 2001. Fifty years since Lashley's In Search of the Engram: Refutations and conjectures. J Hist Neurosci 10: $308-318$

Colgin LL, Leutgeb S, Jezek K, Leutgeb JK, Moser EI, McNaughton BL, Moser MB. 2010. Attractor-map versus autoassociation based attractor dynamics in the hippocampal network. J Neurophysiol 104: 35-50.

Crick FH. 1979. Thinking about the brain. Sci Am 241: 219-232.

Deisseroth K, Schnitzer MJ. 2013. Engineering approaches to illuminating brain structure and dynamics. Neuron 80: $568-$ 577.

Hassenstein B, Reichardt W. 1956. Systemtheoretische Analyse der Zeit-, Reihenfolgen- und Vorzeichenauswertung bei der Bewegungsperzeption des Ruesselkaufers Chlorophanus. Z Naturforsch 11: 513-524.

Keller PJ, Ahrens MB, Freeman J. 2015. Light-sheet imaging for systems neuroscience. Nat Methods 12: 27-29.

Lister R, Mukamel EA, Nery JR, Urich M, Puddifoot CA, Johnson ND, Lucero J, Huang Y, Dwork AJ, Schultz MD, et al. 2013. Global epigenomic reconfiguration during mammalian brain development. Science 341: 1237905.

Ogawa S, Lee TM, Kay AR, Tank DW. 1990. Brain magnetic resonance imaging with contrast dependent on blood oxygenation. Proc Natl Acad Sci 87: 9868-9872.

Petersen SE, Fox PT, Posner MI, Mintun M, Raichle ME. 1988. Positron emission tomographic studies of the cortical anatomy of single-word processing. Nature 331: 585589 .

Redondo RL, Kim J, Arons AL, Ramirez S, Liu X, Tonegawa S. 2014. Bidirectional switch of the valence associated with a hippocampal contextual memory engram. Nature 513: 426430.

Sejnowski TJ. 2015. Consciousness. Daedalus 144: 123-132.

Sejnowski TJ, Churchland PS, Movshon JA. 2014. Putting big data to good use in neuroscience. Nat Neurosci 17: 14401441.

Wall NR, Wickersham IR, Cetin A, De La Parra M, Callaway EM. 2010. Monosynaptic circuit tracing in vivo through Credependent targeting and complementation of modified rabies virus. Proc Natl Acad Sci 107: 21848-21853.

Zhang F, Wang LP, Boyden ES, Deisseroth K. 2006. Channelrhodopsin-2 and optical control of excitable cells. Nat Methods 3: 785-792. 


\section{$\$_{\mathrm{CSH}}^{\infty}$ Cold Spring Harbor Symposia SYMPOSIA on Quantitative Biology}

\section{Summary: Cognition in 2014}

Terrence J. Sejnowski

Cold Spring Harb Symp Quant Biol 2014 79: 237-241 originally published online May 6, 2015 Access the most recent version at doi:10.1101/sqb.2014.79.024885

References This article cites 15 articles, 3 of which can be accessed free at: http://symposium.cshlp.org/content/79/237.full.html\#ref-list-1

\section{License}

Email Alerting Receive free email alerts when new articles cite this article - sign up in Service the box at the top right corner of the article or click here. 